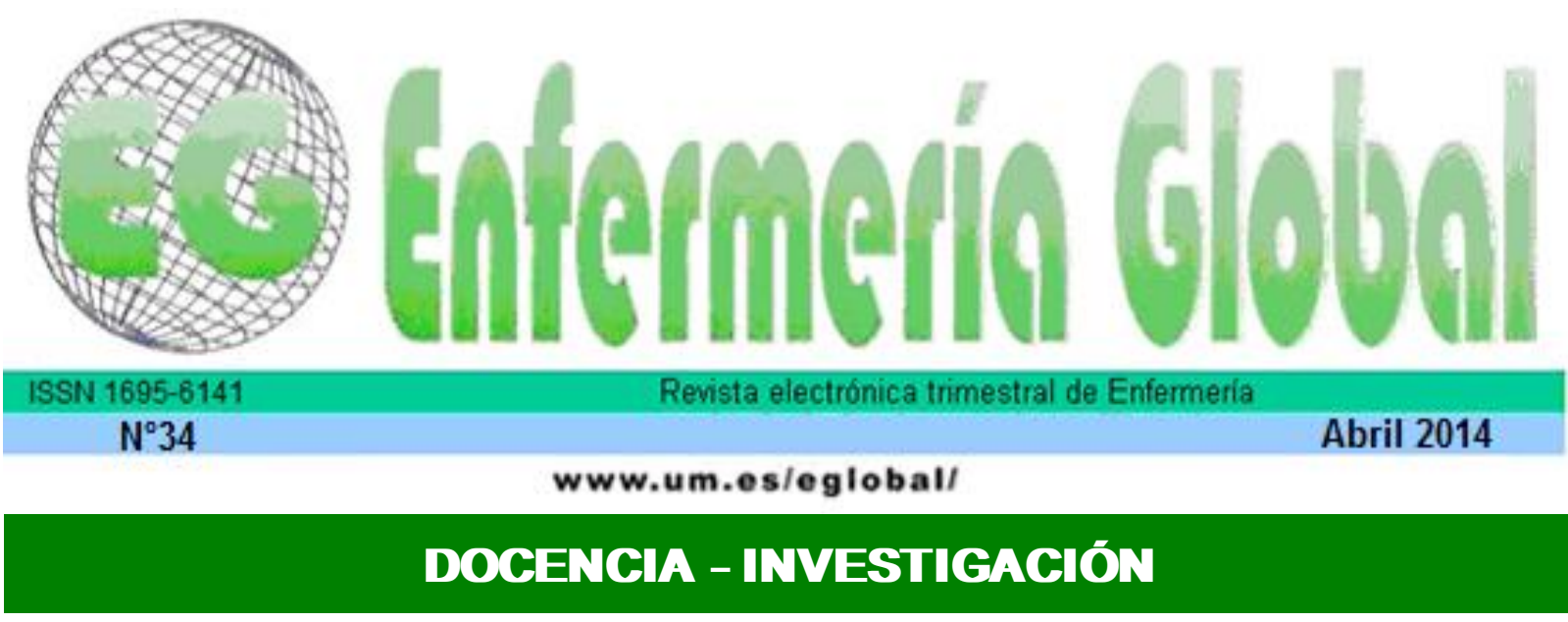

\title{
Vínculos profesionales en el trabajo de enfermería: elemento importante para el cuidado
}

Vínculos profissionais no trabalho da enfermagem: elemento importante para o cuidado

Professional ties in nursing work: important element for care

\section{*Jacondino, Michelle Barboza ${ }^{* *}$ Martins, Caroline Lemos ***Thofehrn, Maira Buss ${ }^{* * * *}$ Garcia, Bianca Lessa ${ }^{* * * * *}$ Fernandes, Helen Nicoletti ${ }^{* * * * * *} J o n e r$, Leandro Rauber}

*Especialista en Educación. Alumna de Máster del Programa de Posgraduación en Enfermería. E-mail: michellejacondino@gmail.com **Especialista en Enfermería del Trabajo. Alumna de Máster del Programa de PosGraduación en Enfermería ***Post doctorado en Enfermería. Profesora Adjunta IV de la Facultad de Enfermería ****Enfermera del Hospital Unimed de Caxias do Sul-RS, Alumna de Máster del Programa de Posgraduación en Enfermería ${ }^{* * * * * E n f e r m e r a . ~ M i e m b r o ~ d e l ~ G r u p o ~ d e ~ i n v e s t i g a c i o ́ n ~}$ NEPEN- Núcleo de Estudios en Prácticas de Salud y Enfermería ${ }^{* * * * *}$ Estudiante del $9^{\circ}$ semestre de la Facultad de Enfermería. Miembro del Grupo de investigación NEPEN. Universidad Federal de Pelotas(UFPel). Brasil.

Palabras clave: Enfermería; Relaciones interpersonales; Equipo de enfermería; Teoría de enfermería.

Palavras chave: Enfermagem; Relações interpessoais; Equipe de enfermagem; Teoria de enfermagem.

Keywords: Nursing; Interpersonal relationships; Nursing staff; Nursing theory.

\section{RESUMEN}

Objetivo: Conocer los vínculos profesionales establecidos en el proceso de trabajo de un equipo de enfermería de una unidad de hospitalización quirúrgica.

Método: Estudio cualitativo, realizado con cinco profesionales de un equipo de enfermería de una unidad de hospitalización quirúrgica, a través de la observación simple no participante y cuestionario autoaplicado. Los datos fueron sometidos a análisis temático y fue utilizado como marco teórico referencial la Teoría de los Vínculos Profesionales. 
Resultados: Los resultados indican vínculos profesionales agregadores y positivos entre los trabajadores de enfermería, lo que favorece el desarrollo del cuidado. El enfermero fue considerado un importante agente social para unir al equipo de enfermería en favor de la atención a la salud y desarrollar las potencialidades de los trabajadores. La integración, la acogida y la unión entre los trabajadores son esenciales para el desempeño del proceso de trabajo en enfermería.

Conclusiones: La Teoría de los Vínculos Profesionales se puede utilizar para fortalecer los vínculos entre el equipo de enfermería en busca de un ambiente saludable de trabajo a partir de relaciones humanas más armónicas.

\section{RESUMO}

Objetivo: conhecer os vínculos profissionais no processo de trabalho de uma equipe de enfermagem.

Método: estudo qualitativo, realizado com cinco profissionais de uma equipe de enfermagem da unidade de internação cirúrgica de um hospital de ensino do Rio Grande do Sul, por meio de observação simples não participante e questionário autoaplicado. Os dados foram submetidos à analise temática e se utilizou como referencial teórico a Teoria dos Vínculos Profissionais.

Resultados: os resultados mostram vínculos profissionais agregadores e positivos entre os trabalhadores de enfermagem, o que favorece ao desenvolvimento do cuidado. $O$ enfermeiro foi considerado agente social importante para unir a equipe de enfermagem em prol da assistência em saúde e desenvolver as potencialidades dos trabalhadores. A integração, o acolhimento e a união entre os trabalhadores são essenciais para o desempenho do processo de trabalho em enfermagem.

Conclusão: a Teoria dos vínculos profissionais pode ser utilizada para fortalecer os vínculos entre a equipe de enfermagem em busca de ambiente de trabalho saudável a partir de relações humanas mais harmoniosas.

\section{ABSTRACT}

Objective: This study aims to know the professional ties established in the work process of a nursing staff of a surgical admission unit.

Method: It is a qualitative study, conducted with five professionals of a nursing staff of a surgical admission unit, through a non-participant simple observation and self-applied questionnaire. The data were submitted to thematic analysis and the Theory of Professional Ties was used as the theoretical reference.

Results: The results indicate aggregator and positive professional ties among nursing workers that favor the development of care. The nurse was considered an important social agent to join the nursing staff in favor of health attendance and to develop the capacities of workers. Integration, acceptance and union among workers are essential to the performance of work process in nursing.

Conclusions: The Theory of Professional Ties can be used to strength ties among the nursing staff, in search of a healthy work environment from human relations more harmonious.

\section{INTRODUCCIÓN}

En el proceso de trabajo de enfermería, por cuestiones de división social y técnica del trabajo, los profesionales realizan el cuidado fragmentado y, por tanto, existe la necesidad de comunicación y articulación de estos trabajadores en pro de la prestación de la asistencia a los individuos que necesitan de cuidados ${ }^{1}$. Con todo, se sabe que las relaciones interpersonales en el equipo sanitario, así como en enfermería, son referidas como factor contributivo al estrés oriundo del ambiente donde se desarrollan las actividades laborales ${ }^{2}$. 
En el conjunto de las acciones de salud es preciso considerar que el proceso de trabajo en enfermería es complementario e interdependiente, influyendo y estando influido en todo momento por los miembros del equipo de enfermería ${ }^{3}$. Así, el trabajo en equipo en enfermería es fundamental para el desarrollo de la práctica asistencial, y el fortalecimiento de este equipo por medio del establecimiento de vínculos profesionales, reconocimiento del trabajo del otro y la colaboración entre las personas son esenciales para la puesta en marcha del cuidado ${ }^{4}$. Sin embargo, las instituciones asistenciales no permiten una reflexión sobre el proceso de trabajo que especifica que es el asistir en salud, así como tampoco adhieren la participación de los trabajadores en la organización del trabajo sanitario y pierden la cualificacióndel cuidado ${ }^{5}$.

De esta forma, se evidencia que el enfermero puede tomar la iniciativa en la organización del trabajo en salud y elaboración de la planificación de acciones de los profesionales teniendo como foco el establecimiento de vínculos profesionales, los cuales pueden ser comprendidos como vínculos afectivos y sociales, con énfasis en la interrelación. Así, la motivación, el compromiso y el dinamismo son elementos importantes para agregar al equipo y estimular relaciones interpersonales saludables ${ }^{3}$.

En esta perspectiva, es interesante movilizar al equipo de enfermería para la transformación del cotidiano de trabajo, en el cual se establezcan relaciones humanas más armoniosas, basadas en el respeto mutuo y cooperación entre los miembros del equipo, teniendo en cuenta que para la realización del trabajo en enfermería es fundamental la cooperación y el trabajo en grupo ${ }^{6}$.

Frente a ese contexto, la Teoría de los Vínculos Profesionales (TVP) surge como propuesta a partir de la teorización de las relaciones establecidas en el ambiente de trabajo en enfermería, por medio del fortalecimiento del equipo de enfermería, mediada por la formación y afirmación de vínculos profesionales. De este modo, la TVP corresponde a un conjunto de definiciones y estrategias generales, flexibles, interdependientes y puede ser considerada una referencia conceptual y práctica para el desarrollo del trabajo en enfermería ${ }^{7}$.

A partir de esto, se comprende que el establecimiento y afirmación de vínculos profesionales saludables posibilita la promoción de un ambiente de trabajo agradable, e implica positivamente en la finalidad del trabajo de enfermería, en la transformación del cuerpo del ser humano a partir del cuidado prestado ${ }^{8,5}$.

Así, la TVP puede ayudar a los trabajadores en el desarrollo de la práctica profesional de enfermería, al posibilitar la reflexión de los profesionales frente a su trabajo y las relaciones sociales establecidas en el ambiente laboral.

Ante lo expuesto, este estudio tiene por objetivo conocer los vínculos profesionales en el proceso de trabajo de un equipo de enfermería.

\section{METODOLOGÍA}

Estudio exploratorio y descriptivo de naturaleza cualitativa.

Este estudio es un recorte del proyecto de investigación "Implantación y evaluación de la viabilidad de la Teoría de los Vínculos Profesionales en la práctica de la enfermería". El proyecto atendió a las recomendaciones para investigaciones que involucran seres humanos ${ }^{9}$ y fue aprobado por el Comité de Ética en Investigación de 
la Facultad de Medicina de la Universidad Federal de Pelotas (protocolo número 060/2008).

Los datos fueron colectados en el mes de mayo de 2011, en dos etapas. La primera fue realizada mediante la observación simple y no participante de un turno de trabajo de un equipo de enfermería. Los profesionales, después de orientados acerca de los objetivos y metodología de la investigación, aceptaron participar en la investigación por medio de la firma del término de consentimiento libre y esclarecido. La observación tuvo como finalidad observar el proceso de trabajo del equipo de enfermería, con enfoque en los vínculos profesionales entre los miembros del equipo de enfermería, los cuales fueron registrados por las investigadoras en un diario de campo. La segunda etapa estuvo compuesta por un cuestionario autoaplicado con los participantes de la primera etapa de la investigación.

La investigación adoptó el cuestionario autoaplicado en virtud de la solicitud de los propios participantes, pues estos sujetos se encuentran asignados a un hospital de enseñanza y forman parte de diversos estudios en el transcurso del año, los cuales a veces no disponen de tiempo para otra modalidad de colecta de datos. De este modo, se adoptó esa forma de colectar las informaciones para alcanzar el objetivo propuesto en esta investigación.

Respondieron el estudio cinco trabajadores del equipo de enfermería, siendo uno enfermero y cuatro técnicos de enfermería. Para asegurar el anonimato de los participantes, los profesionales fueron identificados por medio de la letra "E", correspondiente a la enfermería, seguida de una numeración derivada de la secuencia de la aplicación de los cuestionarios.

Los datos obtenidos en esta investigación se sometieron al analisis temático ${ }^{10}$ conforme los pasos operacionales preconizados: la ordenación de los datos, la clasificación de los datos y el análisis final. La ordenación de los datos es la asignación de las entrevistas realizadas con el equipo de enfermería. Después, se procedió a la clasificación de los datos por medio de lectura fluctuante para identificar ideas centrales y estructuras de relevancia, las cuales pudiesen indicar la base de confronto del material empírico con los estudios existentes sobre el asunto propuesto. La clasificación permitió visualizar las partes que componen el todo del trabajo del equipo de enfermería, sin embargo para realizar la interpretación y el análisis final fue necesrio recomponer el todo y analizar esas relaciones en su conjunto, de manera complementaria e interdependiente ${ }^{10}$.

Ante esto, se establecieron tres temas para análisis titulados: a) Interacciones humanas en el trabajo de enfermería y su implicación en la producción de cuidados b) Cooperación como componente agregador entre los trabajadores de enfermería c) Compromiso en el trabajo: factor essencial para todos los involucrados en el trabajo de enfermería.

Para la interpretación de los resultados obtenidos se utilizó como referencial teórico la Teoría de los Vínculos Profesionales ${ }^{6}$. 


\section{RESULTADOS Y DISCUSIÓN}

\section{Interacciones humanas en el trabajo de enfermería y su implicación en la producción de cuidados}

El trabajo sanitario es amplio y de múltiples dimensiones, constituído por una red de relaciones e interacciones en la cual se encuentra el individuo ${ }^{11}$. El trabajo en salud está constituído por diferentes núcleos profesionales, cada cual con su formación y prácticas de trabajo, pero que tienen un lazo de unión que es el usuario del servicio de salud a quien se prestará este trabajo ${ }^{12}$.

De esta manera, es relevante considerar que la subjetividad es parte del trabajo en salud, pues el objeto de trabajo es el elemento humano y, por tanto, a pesar de haber intervenciones técnicas, estas están siempre acompañadas de relaciones interpersonales ${ }^{13}$.

La enfermería integra la prestación de servicios sanitarios y desarrolla parte del trabajo en salud, que tiende como producto final a la prestación de la asistencia ${ }^{14,5}$. Así, el trabajo de enfermería es considerado un trabajo inmaterial, pues no da origen a productos que se puedan estocar o comercializar, y sin servicios que son consumidos y producidos en el momento de su realización, en el preciso momento de la prestación de la asistencia ${ }^{5}$.

La tarea profesional del trabajo de enfermería es el cuidado terapéutico entendido como toda y cualquiera acción que el profesional desarrolla junto al ser humano, y debe ocurrir a partir de un autoconocimiento en cuanto ser trabajador ${ }^{3}$. A partir de los comentarios de los participantes de la investigación se desprende que es la propia tarea profesional la que une al equipo para la operatividad del cuidado. Los trabajadores perciben que la interacción en el grupo es favorable y conveniente para la realización del trabajo, al destacar el compañerismo y la amistad como factores positivos en el cumplimiento de la tarea, así como los lazos de compañerismo y confianza. En la observación siguiente se refuerza este aspecto.

El equipo de enfermería trabaja todo el tiempo en la prestación de la asistencia del ser humano. En muchos momentos de la prestación del cuidado el equipo se ayuda en la realización de las tareas, preparación de la medicación, baño y curativos. Observación.

La integración del equipo de enfermería es un proceso social que tiende a unificar el equipo y a transcender la individualidad de sus miembros, considerando que cada grupo de trabajo posee dinámica y movimiento ${ }^{6}$. En esta investigación se observó que a pesar de que los trabajadores estaban sometidos al proceso de trabajo fragmentado y rutinario en el ambiente hospitalario, en función de la organización capitalista del trabajo y sus implicaciones, consiguen desdoblar las dificultades encontradas con la cooperación mutua y confianza entre el equipo, al utilizar la tarea profesional como punto de convergencia entre los miembros del grupo.

En la presente investigación, se nota la articulación entre los miembros del equipo de enfermería en dirección a una finalidad específica y los vínculos profesionales en el trabajo de la enfermería son elementos importantes para la realización del cuidado. De este modo, es posible que la construcción de vínculos durante la jornada asistencial, esté aliado al deseo de prestar atención en salud más humanizada y 
competente, al unir al equipo en pro del usuario que necesita de cuidados. Las declaraciones que siguen refuerzan este apunte.

"El grupo trabaja bien integrado y articulado en pro del bienestar del cliente y creo bien conveniente y provechoso". E.1

"Recibo la acogida de mis colegas y apoyo siempre que preciso. Esto ayuda a desarrollar el trabajo". E. 2

La interacción de los profesionales, en este estudio, se traduce como acogida y esta atención acogedora ocurre para la cooperación y compromiso en el trabajo. La TVP enfatiza el autoconocimiento como componente indispensable para la consolidación de vínculos profesionales en el trabajo, siendo primordial la aceptación de uno mismo, para la posterior aceptación del otro. Así, el ser humano, al sentirse seguro, empapa este sentimiento en las relaciones con los otros, siendo posible tornar el ambiente institucional en un lugar de realizaciones, de crecimiento personal y de interacción con el colectivo ${ }^{3}$. En este sentido, los vínculos profesionales son dispositivos esenciales en el entramado de un proceso de trabajo en salud parcelado, en busca de un grupo de trabajo más articulado.

Otro elemento esencial para sentirse acogido e integrado en el grupo está directamente interconectado a las relaciones horizontales en el trabajo, ratificando la importancia de la disolución de la jerarquía profesional entre enfermeros y técnicos de enfermería. Una de los participantes de la investigación enfatiza esta cuestión:

"Considero un equipo de trabajo unido, el que acoge a todos sin jerarquía entre enfermeros y técnicos de enfermería, lo que es importante para realizar el trabajo. Me siento integrada en el grupo aqui" E.4

La división de tareas y organización del trabajo en enfermería por el enfermero es una cuestión histórica y social, pues este profesional se ha convertido en el coordinador de las actividades de los miembros del equipo de enfermería y, no raramente, de los demás trabajadores del equipo de salud ${ }^{11}$. El enfermero instaura relación de superioridad sobre auxiliares y técnicos de enfermería, con poderes que lo diferencian de estos profesionales ${ }^{15}$, estando estos respaldados por la ley del ejercicio profesional n. .97 .498 , de 25 de junio de $1986^{16}$, que determina ser privativo del enfermero aspectos como administrar y coordinar la unidad de enfermería y los conocimientos relativos a la asistencia en enfermería. Con todo, es preciso tener claro que la imposición del poder de forma verticalizada en el proceso de trabajo de enfermería puede provocar opresión en los trabajadores.

De este modo, el enfermero es percibido como coordinador del equipo, un líder en la organización de la implementación de la asistencia, al involucrar a los diferentes profesionales en el cuidado. Además, se espera que el enfermero vincule al equipo de enfermería en el desarrollo del trabajo de salud, donde todos tejen una red de cuidado, incluso de forma parcelada, pues es preciso tener claro que el objeto de trabajo es común a todos.

Así, es el enfermero quien organiza el proceso de trabajo de la enfermería, divide las tareas a desempeñar por los técnicos de enfermería y coordina las acciones para la salud del equipo de enfermería en pro del usuario ${ }^{17}$. En este contexto, cabe al coordinador mantener relación armónica entre los trabajadores, a partir del diálogo y 
la reflexión acerca de las relaciones humanas entre los miembros del equipo y la relación del grupo con la tarea profesional ${ }^{3}$.

De esta manera, la postura adoptada por el enfermero reflexiona sobre todo el proceso de enfermería, lo que afecta a la calidad de las relaciones humanas para la prestación del cuidado.

\section{La cooperación como elemento agregador entre los trabajadores de enfermería}

La teoria de los vínculos profesionales postula que la busca de cuidado terapéutico de calidad debe iniciar en la atención a los colaboradores que componen el equipo de enfermería, en esa perspectiva compartida de la idea de que el equipo es la nomenclatura establecida para todo agrupamiento de personas que desempeñan una actividad profesional ${ }^{18}$. Al considerar lo anterior se evidencia que para la TVP se espera más de los colaboradores, con miras a alcanzar su objetivo y, por esta razón, la Teoría adopta el concepto de grupo presentado por Pichon-Rivière ${ }^{19}$. Se entiende por grupo un conjunto delimitado de personas que tienen en común la vivencia en un mismo espacio y un mismo tiempo, teniendo el propósito de ejecutar determinadas tareas comunes para el grupo, a fin de alcanzar los objetivos del todo, ese grupo está articulado por representación interna, que en enfermería se remite al enfermero. De este modo, la tarea es el elemento organizador de los procesos de pensamiento, comunicación y acción que ocurren en el grupo ${ }^{19}$.

\section{"Estamos todos articulados en el cumplimiento del trabajo y mantenemos buenos lazos de amistad y compañerismo". E.2}

De esta manera, se entiende que para la formación de un grupo de trabajo debe haber reflexión y comprensión por parte del equipo de enfermería y, para esto, el papel del enfermero es esencial, pues es un profesional que asume el liderazgo y coordinación del trabajo asistencial de salud. Con todo, algunas variables pueden contribuir o no al alcance de las finalidades del grupo $y$, por tanto, es preciso considerar los determinantes que se encuentran articulados al éxito o fracaso del alcance de la tarea grupal. Los determinantes pueden estar ligados a aspectos estructurales, de funcionamiento, de dinámica y de interrelaciones, y, de este modo el éxito del trabajo parte de la concienciación sobre la existencia de estos determinantes, que pueden ser visualizados en la medida en que el grupo introyecta reflexiones teóricas y práticas del trabajo grupal ${ }^{20}$.

De esta forma, la teoría de los vínculos profesionales puede ayudar en las reflexiones teóricas del grupo, pues se considera un modelo de gestión para el trabajo en equipo de enfermería, entendida como herramienta mediadora que considera la subjetividad de los trabajadores, con miras a minimizar las dificultades vivenciadas, en lo que se refiere a las relacionees humanas en el trabajo ${ }^{21}$. Los profesionales de enfermería al ejecutar su trabajo en ambiente agradable desarrollan sus actividades con placer y satisfacción, repercutiendo así en la calidad del cuidado terapéutico ${ }^{6}$. En este sentido, los profesionales de enfermería muestran aspectos de cooperación y unión como formas de integrar al equipo y facilitar el desarrollo del cuidado.

"Uno de los aspectos facilitadores del trabajo es la disponibilidad de ayuda mutua, la cooperación entre los funcionarios". E.3 
La vida en grupo debe oportunizar un universo de experiencias y confianza entre las personas, contribuyendo al desarrollo y crecimiento de los profesionales de enfermería, por medio del descubrimiento de sí mismo y de los otros miembros del equipo ${ }^{6}$.

El enfermero es el elemento de referencia para los demás componentes del equipo de enfermería, pues en él el equipo encuentra su punto de apoyo, siendo percibido como facilitador de proyectos y deseos de los trabajadores, estimulador para la formación de vínculos profesionales saludables, del desarrollo pleno del potencial de todos, lo que puede influir en la calidad de la asistencia ${ }^{22}$. De este modo, las potencialidades de los trabajadores pueden ser estimuladas en el trabajo, mediadas por la utilización de la TVP, para construir un grupo cohesivo, reflexivo y crítico, pues es la herramienta mediadora de trabajo.

En ese sentido, una de las alternativas de unir al equipo de enfermería y mantener a los trabajadores actualizados es la formación en servicio, teniendo en cuenta que es una oportunidad de identificar el potencial de cada miembro del equipo. Sin embargo, los participantes de este estudio no refieren la ocurrencia de la formación en servicio, mas perciben esa actividad como sinónimo de trabajo en grupo, una forma de unir al equipo.

“(..) falta una formación en servicio, un tipo de trabajo en grupo que pueda unirnos en el trabajo. Todas las actividades extras realizadas aqui son propuestas por los técnicos y enfermeros y no por la gerencia, por ejemplo, en proporcionar algo diferente, cambios en el sector". E.4

La formación en servicio es un proceso educativo aplicado a las relaciones humanas con la intención de desarrollar las áreas cognitiva, psicomotora y relacional de los profesionales, respaldada por el aprendizaje continuo, dirigido a la mejora de sí mismo, buscando la competencia personal, profesional y social ${ }^{23}$.

El enfermero al utilizar el conocimiento científico actualizado en el servicio de salud contribuye al desarrollo personal y profesional del equipo de trabajo. La formación en servicio ayuda al establecimiento de los vínculos profesionales saludables en el ambiente de trabajo, en la medida en que todos los del grupo visualizan el objetivo común del equipo de enfermería. Por eso, se entiende que la Teoría de los Vínculos Profesionales consiste en un conjunto de estrategias para la mejora de las relaciones interpersonales en el trabajo en salud y de esta forma, contribuye a la prestación del cuidado terapéutico.

"Nosotros [equipo de enfermería] no tenemos formación en servicio desde que esta enfermera entró. Antes nosotros teníamos y era bueno" (...)E.2

La Teoría de los Vínculos Profesionales tiene como uno de sus aspectos principales la creación de espacios educativos en el ambiente de trabajo, con estímulo a las acciones de formación en servicio, con miras a promover el crecimiento del profesional de enfermería como ser humano y trabajador, por medio de la actualización y perfeccionamiento del conocimiento, cualificando la asistencia en salud $^{6}$. 
Otro aspecto importante que puede ser considerado relevante por las organizaciones institucionales de salud es la formación continua, que ayuda en la formación de los vínculos profesionales, por medio del perfeccionamiento profesional, supliendo las necesidades de conocimiento, en la constante actualización teórico-práctica, a partir de cursos, capacitaciones y posgraduaciones. El intercambio de experiencias entre el equipo de enfermería puede permitir la consolidación de los vínculos en el trabajo, que además de proporcionar la cualificación de los trabajadores para las actividades profesionales, se torna un factor agregador y positivo para el grupo así como para el objeto de trabajo del proceso de trabajo de enfermería, o sea, el usuario que recibe la asistencia de enfermería.

Los participantes de esta investigación muestran que los cambios realizados en el trabajo son fruto de la voluntad de los enfermeros y técnicos en cualificar a su personal, pues no hay interés por parte de la administración institucional en proporcionar formación en servicio al equipo de enfermería. En las declaraciones de los participantes se evidencia la percepción de los profesionales de enfermería acerca de la necesidad de estar en constante cualificación para efectuar el cuidado, y se comprende la corresponsabilidad de la institución y también de la gerencia del hospital para la realización y mantenimiento de programas de formación en servicio.

\section{Compromiso en el trabajo: factor esencial para todos los involucrados en el trabajo en salud}

En el trabajo en salud, cada integrante del equipo posee un saber, una formación específica y, con eso, la tendencia de los trabajadores es la de no considerar tales diferencias y trabajar de forma aislada y $\operatorname{solos}^{24}$, lo que implica desencuentros y acciones desarticuladas en la producción en salud. Con todo, el trabajo es un espacio de transformaciones posibles, pues al trabajar el hombre modifica la naturaleza, mas es también modificado por ella en una relación recíproca de doble transformación ${ }^{25}$. Las relaciones sociales y múltiples determinaciones que se constituyen en ese proceso acarrean cambios continuos en la forma de trabajar de los profesionales, las cuales pueden tornar las relaciones humanas en más armós.

En ese contexto, los vínculos profesionales establecidos en el ambiente hospitalario son una estrategia de acción, en la dirección de un trabajo taylorizado, en el que se procucen más divisiones que puntos de unión en los trabajadores, que aunque comparten el mismo espacio, de ritmo y rutinas de trabajo, la propia estructura organizacional, lleva a una confrontación entre los trabajadores, individualmente y en soledad, sometidos a la violencia de la productividad ${ }^{26}$. Todavía es significativo enfatizar que los profesionales de salud son todos trabajadores del cuidado, cada uno en su dimensión, mas siempre actuando en pro del usuario ${ }^{27}$. En esa coyuntura de trabajo en salud, el enfermero, además de ser mediador del trabajo de enfermería y del cuidado terapéutico puede ser reconocido como sujeto importante para el desarrollo personal y profesional de los miembros del equipo de enfermería.

"Como una de las integrantes más nueva del equipo de enfermería, percibo que está ocurriendo la adaptación necesaria para que yo pueda desarrollar mis potencialidades en el trabajo y eso es bueno". E.2

"Las relaciones interpersonales en la unidad son de confianza y respeto mutuo, y esto facilita el desarrollo de la actividad profesional". E.3 
"En la unidad ocurre el acuerdo entre el equipo de enfermería, lo que es necesario para que yo pueda desarrollar mis potencialidades en el trabajo". E.5

El proceso de trabajo de enfermería puede entenderse como la transformación del ser humano que necesita de cuidado por medio de la intervención del equipo de enfermería, que utiliza herramientas de trabajo para realizar tal cambio. Los profesionales que trabajan en el área de la salud necesitan participar en continuas capacitaciones para desarrollar sus potencias y habilidades para entonces efectuar la actividad profesional. Destaca el que estas capacitaciones deben comenzar por las tecnologías leves, que abarcan la acogida, responsabilización, así como la formación de vínculos entre profesionales y usuarios, teniendo en cuenta que son elementos básicos y esenciales en la realización del cuidado y administración de las relaciones humanas en el contexto laboral ${ }^{27}$.

"Yo creo que aqui, los enfermeros nos transmiten mucha seguridad, conocimiento y experiencia, y esto hace que junto con el resto del equipo, yo pueda mejorar mi desarrollo prpofesional". E.2

En este sentido, el enfermero se presenta como ser participativo, cooperativo y comprometido con el equipo de enfermería, al respetar la individualidad de cada uno, mantener el consenso de las decisiones del grupo y utilizar la creatividad en pro de la calidad en la realización del cuidado terapéutico ${ }^{6}$. El enfermero es reconocido como persona capaz de identificar las aptitudes, desarrollar habilidades y los talentos de cada miembro del equipo.

Así, el enfermero al adoptar postura de mantenimiento de diálogo compartido, transparente y planificación colectiva estará contribuyendo al establecimiento de vínculos profesionales saludables entre los miembros de su equipo, por medio de la participación de todos los integrantes en las tomas de decisiones y organización del trabajo. Además, este profesional promueve la valorización de los actores sociales involucrados en el trabajo en salud, siendo que estos se reconocen como parte esencial para el éxito de las actividades.

\section{CONSIDERACIONES FINALES}

Los resultados de este estudio mostraron que los vínculos profesionales agregadores y positivos entre los trabajadores de enfermería favorecen el desarrollo del cuidado, pues contribuyen a que los miembros del equipo de enfermería se sientan parte integrante de la organización y planificación de las actividades en salud. En ese sentido, los vínculos son un elemento importante que puede proporcionar calidad en la asistencia en salud, por el hecho de la participación proactiva de todos en la decisión de las prácticas de trabajo que atraviesan el ambiente hospitalario.

Es imprescindible el desarrollo de práctica de trabajo articuladora, comunicativa y de diálogo abierto entre los trabajadores de enfermería, dirigida al entendimiento mutuo y cooperativo en el trabajo, pues apostamos que la participación de los profesionales es uno de los muelles propulsores para posibles transformaciones del proceso de trabajo de enfermería.

En este estudio, se observó que el enfermero fue percibido por el equipo de enfermería como coordinador del grupo de trabajo, pues, para que el cuidado 
terapéutico tenga lugar este profesional necesita mediar las relaciones humanas entre equipo de enfermería, y también entre equipo de salud y las gerencias de la institución hospitalaria, para alcanzar las necesidades biopsicosociales de los usuarios.

El enfermero fue considerado agente social importante para unir al equipo de enfermería en pro de la asistencia sanitaria y desarrollar las potencialidades de estos trabajadores, dado que fue señalado como un facilitador, orientador y motivador para la afirmación de vínculos profesionales, además de ser el responsable de la división y organización de las actividades realizadas por el equipo. Acreditamos que la planificación participativa, la unión y la acogida entre los miembros del equipo de enfermería son aspectos que contribuyen a un ambiente de trabajo más saludable por medio de vínculos profesionales saludables. En este contexto, entendemos que la TVP puede ser utilizada en enfermería como herramienta mediadora para la práctica profesional, considerándose un instrumento de trabajo relevante, al tender a la formación de vínculos profesionales saludables, al fortalecimiento del equipo de enfermería, implicando ciertamente en la cualificación del cuidado.

\section{REFERENCIAS}

1 Hausmann M, Peduzzi M. Articulação entre as dimensões gerencial e assistencial do processo de trabalho do enfermeiro. Texto Contexto Enfermagem; 2009. 258 p.

2 Jorge LLS, Enirtes CPM. Estresse e implicações para o trabalhador de enfermagem. Informe-se em promoção da saúde; 2006. 16 p.

3 Thofehrn Maira Buss, Leopardi Maria Tereza. Teoria dos vínculos profissionais: um novo modo de gestão em enfermagem. Texto contexto - enferm. [serial on the Internet]. 2006 Sep [cited 2012 Dec 02] ; 15(3): 409-417.

4 Souza SS, Costa R, Shiroma LMB, Maliska ICA, Aamadigi FR, Pires DEP, Ramos FRS. Reflexões de profissionais de saúde acerca do seu processo de trabalho. Rev. Eletrônica de Enfermagem; 2010. 449 p.

5 Pires D. Reestruturação produtiva e trabalho em saúde no Brasil. São Paulo: Annablume; 2008.

6 Thofehrn MB, Leopardi MT. Teoria dos vínculos profissionais: formação de grupo de trabalho. Pelotas (RS): Ed. universitária UFPel; 2009.

7 Thofehrn MB, Leopardi MT. Teoria dos vínculos profissionais modo de gestão em enfermagem. Texto Contexto Enfermagem; 2006. 409p.

8 Thofehrn MB, Meincke SMK, Soares MC, Heck RM. Organizadores. Práticas de gestão e gerenciamento no processo de trabalho em saúde. Pelotas (RS): Ed.universitária UFPel; 2009.

9 Ministério da saúde. Conselho Nacional de Saúde. Comissão Nacional de Ética em Pesquisa. Resolução $n^{\circ} 196$ de 10 de outubro de 1996: diretrizes e normas regulamentadoras de pesquisa envolvendo seres humanos. Brasília, 1996.

10 Minayo MCS. O desafio do conhecimento: pesquisa qualitativa em saúde. $12^{\mathrm{a}}$ ed. São Paulo (SP): Hucitec, 2010.

11 Magda SK, Dirce SB, Helenice MS, Marilene LW, Andréa MV, Maria TZ, Vera R, Evanguelia KAS. Tecnologias de cuidado em saúde e enfermagem e suas perspectivas filosóficas. Texto Contexto Enfermagem; 2006. 178p.

12 Judite HB, Denise EPP, Flávia RSR. Dimensões do trabalho da enfermagem em múltiplos cenários institucionais. Tempus - Actas de Saúde Coletiva - O Trabalho em Saúde, 2011. 
13 Pires DEP. A estrutura objetiva do trabalho em saúde. In: Leopardi MT. Processo de trabalho em saúde: organização e subjetividade. Florianópolis: Papa-Livros; 1999. $64 p$.

14 Felli VEA, Peduzzi M. O trabalho Gerencial em enfermagem. In: Kurcgant, P. (Org). Gerenciamento em enfermagem. 2. Ed. Rio de Janeiro: Guanabra Koogan; 2012. $113 \mathrm{p}$.

15 Lima MADS, Almeida MCPO. Trabalho de enfermagem na produção de cuidados de saúde no modelo clínico. Rev. gaúcha Enfermagem; 1999. 86p.

16 Governo Federal (BR). Lei № 7.498/86, de 25 de Junho de 1986 - Dispõe sobre a regulamentação do exercício da Enfermagem e dá outras providências. Brasília (DF): Governo Federal, 1986.

17 Francisco CPR. O Trabalho do enfermeiro em unidades de internação de um hospital geral da 14aㅡ CRS do Rio Grande do Sul. (dissertação). Porto Alegre (RS): Universidade Federal do Rio Grande do Sul/UFRGS; 2003.

18 Bueno, JGS. A produção acadêmica sobre inclusão escolar e Educação Inclusiva. En E.G. Mendes, M.A. Almeida, M.C.P.I. Hayashi (Org.), Temas em Educação Especial: Conhecimentos para fundamentar a prática. Araraquara: Junqueira \& Marin; 2008. 31p.

19 Pichon-Reviére E. O processo grupal. 6ed. Rev. São Paulo: Martins Fontes, 1998. 20 Câmara MFB, Damásio VF, Munardi DB. Vivenciando os desafios do trabalho em grupo. Rev Eletr Enf [Internet]; 2008 [acesso em: 3 jun.2012]. Disponível em: http://www.fen.ufg.br/revista/v10/n3/v10n3aXX.htm

21 Thofehrn MB, Amestoy SC, Porto AR, Arrieira IC, Dal Pai D. A dimensão da subjetividade no processo de trabalho da enfermagem. Rev. Enfermagem. Saúde; 2011. 190p.

22 Gelbcke FL, Souza LA, Dal Sasso GM, Nascimento E, Bulb MBC. Liderança em ambientes de cuidados críticos: reflexões e desafios à Enfermagem Brasileira. Revista Brasileira de Enfermagem; 2009.136p.

23 Paschoal AS, Mantovani MF, Méier MJ. Percepção da educação permanente, continuada e em serviço para enfermeiros de um hospital de ensino. Rev Esc Enfermagem USP; 2007. 478p.

24 Fortuna CM. Et al. O trabalho de equipe no Programa de Saúde de Família: reflexões a partir de conceitos do processo grupal e de grupos operativos. Rev. Latino Americano; 2005. 262p.

25 Marx, K. O capital: Crítica da economia política. 29. Ed. Rio de Janeiro: Civilização Brasileira, tradução Reinaldo Sant'anna; 2011.

26 Dejours, C. A loucura do trabalho: Estudo da Psicopatologia do trabalho. 5 $5^{\text {a }}$ ed. São Paulo: Cortez; 2011.

27 Merhy EE, Franco T. Por uma Composição Técnica do Trabalho Centrada nas Tecnologias Leves e no Campo Relacional in Saúde em Debate; 2003.

ISSN 1695-6141

๑ COPYRIGHT Servicio de Publicaciones - Universidad de Murcia 